\title{
THE SIGNATURE AND ARITHMETIC GENUS OF CERTAIN ASPHERICAL MANIFOLDS
}

\author{
F. THOMAS FARRELL ${ }^{1}$
}

\begin{abstract}
In this paper we show that the signature and arithmetic genus of certain aspherical manifolds $M$ vanish when the center of $\pi_{1} M$ is nontrivial. We make the possibly technical assumption that $\pi_{1} M$ is residually finite.
\end{abstract}

0. Introduction. Gottlieb [4] has shown that the Euler characteristic of a finite, aspherical polyhedron $X$ vanishes, provided $\pi_{1} X$ has a nontrivial center. (Recall that $X$ is aspherical, if $\pi_{i} X$ vanishes for all $i \neq 1$.)

In this note, we show that the signature of a closed, smooth, aspherical manifold $M$ vanishes, provided $\pi_{1} M$ has a nontrivial center and is residually finite. (A group $\Gamma$ is residually finite if, for each $\gamma \in \Gamma$, there exists a subgroup $\Delta_{\gamma}$ with finite index such that $\gamma \notin \Delta_{\gamma}$.) We also prove an analogous result for the arithmetic genus (and the generalized arithmetic genus) of a Kaehler manifold.

The residually finite condition is possibly superfluous, but the author so far has been unable to remove it. (Stallings' paper [7] may be helpful here.) In any event, Malcev [6, Theorem VII] has given the following useful criterion for residual finiteness. Namely $\Gamma$ is residually finite if, for each $\gamma \in \Gamma$, there exists a representation $\varphi: \Gamma \rightarrow \operatorname{GL}(n, \mathbf{R})$ (where $n$ can vary with $\gamma$ ) such that $\varphi(\gamma)$ is not the identity matrix.

I wish to thank Robert Hunter and especially Wu-chung Hsiang for many helpful conversations during the preparation of this paper.

1. The signature. We begin by paraphrasing a basic result from [4].

Corollary [4, I.14]. Let $X$ be a finite, aspherical complex and $\alpha$ an element in the center of $\pi_{1}\left(X, x_{0}\right)$; then there exists a homotopy $h: X \times[0,1] \rightarrow X$ such that

$1^{\circ} . h(x, 0)=h(x, 1)=x$ for all $x \in X$, and

$2^{\circ}$. the closed path $h\left(x_{0}, t\right)$ represents $\alpha$.

This has the following immediate consequence.

LEMMA 1.1. Let $X$ be a finite, aspherical complex such that $\pi_{1} X$ is residually finite and contains a nontrivial center; then there exists a connected, finite-sheeted,

Received by the editors June 16, 1975.

AMS (MOS) subject classifications (1970). Primary 57D20, 58G10; Secondary 53C55.

Key words and phrases. Covering space, Kaehler manifold, residually finite, center.

${ }^{1}$ Research supported in part by NSF grant MPS74-06579. 
regular covering space $f: \bar{X} \rightarrow X$ and a covering transformation $T: \bar{X} \rightarrow \bar{X}$ such that $T$ is homotopic to the identity map but different from it.

Proof. Let $\alpha$ be a nontrivial element in the center of $\pi_{1}\left(X, x_{0}\right), h$ the homotopy posited in Corollary I.14 of [4], and $\Gamma$ a normal subgroup of $\pi_{1}\left(X, x_{0}\right)$ with finite index such that $\alpha \notin \Gamma$. Then $f: \bar{X} \rightarrow X$ is the regular covering space corresponding to $\Gamma$ and $T$ is obtained as follows. Lift $h$ to a homotopy $\bar{h}: \bar{X} \times[0,1] \rightarrow \bar{X}$ such that $\bar{h}(x, 0)=x$ for all $x \in \bar{X}$, and define $T$ by the equation $T(x)=\bar{h}(x, 1)$.

Let $M^{4 n}$ be a connected, closed, smooth, oriented manifold of dimension $4 n$, then the cup-product pairing evaluated on the orientation class of $M$ defines a symmetric, nondegenerate, bilinear form $B$ on $H^{2 n}(M, \mathbf{R})$. Split $H^{2 n}(M, \mathbf{R})$ as the direct sum of two subspaces $H^{2 n}(M, \mathbf{R})=H^{+} \oplus H^{-}$so that $B$ is positive definition on $H^{+}$and negative definite on $H^{-}$, and recall that the signature of $M, \operatorname{Sign}(M)$, is defined by

$$
\operatorname{Sign}(M)=\operatorname{dim} H^{+}-\operatorname{dim} H^{-} .
$$

In addition, if $T: M \rightarrow M$ generates a finite group of orientation preserving diffeomorphisms, then $\mathrm{H}^{+}$and $\mathrm{H}^{-}$can be constructed to be invariant under the automorphism $T^{*}$ of $H^{2 n}(M, \mathbf{R})$ induced by $T$. In this case, $\operatorname{Sign}(T, M)$ is defined by

$$
\operatorname{Sign}(T, M)=\operatorname{Trace}\left(T^{*} / H^{+}\right)-\operatorname{Trace}\left(T^{*} / H^{-}\right) .
$$

TheOREM 1.2. Let $M^{4 n}$ be a closed, smooth, aspherical manifold, then $\operatorname{Sign}(M)$ vanishes provided $\pi_{1} M$ is residually finite and contains a nontrivial center.

Proof. Let $f: \bar{M} \rightarrow M$ and $T: \bar{M} \rightarrow \bar{M}$ be the covering space and transformation posited in 1.1, and $m$ denote the number of sheets of $f$. Since $f$ is a degree $m$, codimension-0 immersion, the Hirzebruch signature theorem [5, Theorem 8.2.2] implies that $\operatorname{Sign}(\bar{M})=m \operatorname{Sign}(M)$. Hence it suffices to show that $\operatorname{Sign}(\bar{M})$ vanishes. By $1.1, T^{*}$ is the identity map, therefore Sign $(\bar{M})=\operatorname{Sign}(T, \bar{M})$. But $T$ has no fixed points, so an application of the Atiyah-Singer $G$-signature theorem [2, Theorem 6.12] yields that $\operatorname{Sign}(T, \bar{M})$ vanishes.

2. The arithmetic genus. We start by recalling the definition of the arithmetic genus. Let $M^{n}$ be a complex analytic manifold of complex dimension $n$, and $A^{k}$ denote the $\mathrm{C}$-module of global smooth $\mathrm{C}$-valued $k$-forms on $M$. Then $A^{k}$ splits as the direct sum $A^{k}=\sum_{p+q=k} A^{p, q}$ where $A^{p, q}$ denotes the $\mathbf{C}$-module of global forms of type $(p, q)$ on $M$. And the exterior derivative $d$ decomposes as $d=\partial+\bar{\partial}$, where $\partial: A^{p, q} \rightarrow A^{p+1, q}$ and $\bar{\partial}: A^{p, q} \rightarrow A^{p, q+1}$. (Here $\partial$ and $\bar{\partial}$ are differentiation with regard to the $z$-variable and $\bar{z}$-variable respectively.) Now for each $p$,

$$
A^{p, 0} \stackrel{\bar{\partial}}{\rightarrow} A^{p, 1} \stackrel{\bar{\partial}}{\rightarrow} \cdots \stackrel{\bar{\partial}}{\rightarrow} A^{p, n}
$$

is a cochain complex whose cohomology we denote by $H^{p, q}$,and the arithmetic genus $\chi(M)$ is given by 


$$
\chi(M)=\sum_{q=0}^{n}(-1)^{q} \operatorname{dim} H^{0, q} .
$$

More generally $\chi^{p}(M)$ is defined by

$$
\chi^{p}(M)=\sum_{q=0}^{n}(-1)^{q} \operatorname{dim} H^{p, q},
$$

and the polynomial $\chi_{y}(M)$, called the generalized arithmetic genus, by

$$
\chi_{y}(M)=\sum_{p=0}^{n} \chi^{p}(M) y^{p} .
$$

THEOREM 2.1. Let $M^{n}$ be an aspherical, closed Kaehler manifold, then $\chi_{y}(M)$ vanishes provided $\pi_{1} M$ is residually finite and contains a nontrivial center.

Remark 2.2. Borel and Hirzebruch have obtained some pertinent calculations of $\chi_{y}(M)$ (hence also $\operatorname{Sign}(M)$ ) for an important class of aspherical, Kaehler manifolds. (See [5, \$22.2] and [3].)

Proof. Let $f: \bar{M} \rightarrow M$ and $T: \bar{M} \rightarrow \bar{M}$ be the covering space and transformation given by 1.1; then the Riemann-Roch theorem [2, Theorem 4.3] implies that $\chi^{p}(\bar{M})=m \chi^{p}(M)$ where $m$ is the number of sheets of $f$. Hence it suffices to show that $\chi^{p}(\bar{M})$ vanishes for $p=0,1, \ldots, n$.

Now the complex analytic map $T$ induces endomorphisms $T^{p, q}$ of $A^{p, q}$ which commute with $\bar{\partial}$ (as well as $d$ ). Denote the induced endomorphism of $H^{p, q}$ by $H^{p, q}(T)$, and define the $p$ th Lefschetz number $L^{p}(T)$ by the formula

$$
L^{p}(T)=\sum_{q=0}^{n}(-1)^{q} \operatorname{trace} H^{p, q}(T) .
$$

Since $T$ has no fixed points, the holomorphic form of the Atiyah-Bott fixed point theorem [1, formula 4.9] implies that $L^{p}(T)=0$. Therefore it remains to show that $\chi^{p}(\bar{M})=L^{p}(T)$, which is a result of the following.

Lemma 2.3. For all $p$ and $q, H^{p, q}(T)$ is the identity endomorphism.

Proof. Use the Kaehler metric on $\bar{M}$ induced by $f$ to define the complex Laplace-Beltrami operator $\square: A^{p, q} \rightarrow A^{p, q}$ and the real Laplace operator $\Delta: A^{k} \rightarrow A^{k}$; then $\Delta=2 \square$. (See [5, pp. 121-124] for more details.) Because $T$ is an isometry, $T^{p, q}$ commutes with $\Delta$, thus also with $\square$, and therefore leaves the kernel of $\square$ invariant. Since ker $\bar{\partial}=\operatorname{ker} \square \oplus$ image $\bar{\partial}$, it suffices to show that $T^{p, q}$ restricted to ker $\square$ is the identity map. But this is a consequence of the Poincare lemma, which says that homotopic maps ( $T$ and the identity) induce chain homotopic maps on the De Rham complex

$$
A^{0} \stackrel{d}{\rightarrow} A^{1} \stackrel{d}{\rightarrow} \cdots \stackrel{d}{\rightarrow} A^{2 n}
$$

together with the equations

$$
\operatorname{ker} d=\operatorname{ker} \Delta \oplus \text { image } d \text { and } \operatorname{ker} \Delta=\operatorname{ker} \square .
$$




\section{REFERENCES}

1. M. F. Atiyah and R. Bott, A Lefschetz fixed point formula for elliptic complexes: II, Ann. of Math. (2) 88 (1968), 451-491. MR 38 \# 731.

2. M. F. Atiyah and I. M. Singer, The index of elliptic operators. III, Ann. of Math. (2) 87 (1968), 546-604. MR 38 \#5245.

3. A. Borel, Compact Clifford-Klein forms of symmetric spaces, Topology 2 (1963), 111-122. MR 26 \#3823.

4. D. H. Gottlieb, A certain subgroup of the fundamental group, Amer. J. Math. 87 (1965), 840-856. MR 32 \#6454.

5. F. Hirzebruch, Topological methods in algebraic geometry, 3rd ed., Die Grundlehren der math. Wissenschaften, Band 131, Springer-Verlag, Berlin and New York, 1966. MR 34 \#2573.

6. A. I. Mal'cev, On the faithful representation of infinite groups by matrices, Mat. Sb. 8 (50) (1940), 405-422; English transl., Amer. Math. Soc. Transl. (2) 45 (1965), 1-18. MR 2, 216.

7. J. Stallings, Centerless groups-an algebraic formulation of Gottlieb's theorem, Topology 4(1965), 129-134. MR 34 \#2666.

Department of Mathematics, Pennsylvania State University, University Park, PennsylVANIA 16802 